\title{
FINASTERIDA: DIEZ AÑOS DE USO CLÍNICO. REVISIÓN SISTEMÁTICA DE LA LITERATURA
}

\author{
J.F. JIMÉNEZ CRUZ*, L. QUECEDO GUTIÉRREZ**, J. DEL LLANO SEÑARÍS** \\ *Servicio de Urología. Hospital Universitario La Fe. Valencia. ${ }^{* * F u n d a c i o ́ n ~ G a s p a r ~ C a s a l . ~ M a d r i d . ~}$
}

Actas Urol Esp. 27 (3): 202-215, 2003

\section{RESUMEN}

"FINASTERIDA: DIEZ AÑOS DE USO CLÍNICO. REVISIÓN SISTEMÁTICA DE LA LITERATURA"

INTRODUCCIÓN: Después de diez años de uso clínico de Finasterida en el tratamiento farmacológico de la Hiperplasia Benigna de Próstata (HBP), nos planteamos revisar la literatura, para analizar aquellos trabajos que por diseño y calidad metodológica, nos permita aplicar recomendaciones basadas en evidencias clínicas.

MATERIAL Y MÉTODOS: Utilizando las bases de datos informáticas disponibles en Medline, Embase, Healthstar, y la Cochrane Library (1990-2002), se recogieron todos los trabajos referentes a pacientes entre 50 y 85 años, con síntomas del tramo urinario inferior sugerentes de HBP y con parámetros objetivos, como flujometría, medición del volumen prostático y del residuo post-miccional, presión del detrusor, efectos adversos, costeefectividad, calidad de vida.

RESULTADOS: Se encontraron un total de 137 referencias, de las cuales 36 cumplen los criterios de inclusión, que permiten su clasificación por niveles de evidencia. Tres tienen nivel I, 12 nivel II, 6 nivel III y 10 nivel IV. Tres son estudios económicos y dos evalúan calidad de vida.

COMENTARIOS: Con alto nivel de evidencia y después de diez años de uso, se demuestra que Finasterida es efectivo en reducir la sintomatología en los pacientes con próstatas mayores de $40 \mathrm{ml}$ y/o PSA >1,4 ngr/ml, con escasos efectos adversos y una clara mejoría en la calidad de vida, por lo que es recomendable con grado $\mathrm{A}$, su uso clínico.

CONCLUSIONES: Finasterida, al reducir el volumen prostático (nivel de evidencia I), modifica la evolución natural de la HBP, disminuyendo el riesgo de retención aguda de orina y de cirugía.

PALABRAS CLAVE: Hiperplasia Benigna de Próstata. Finasterida. Medicina basada en la Evidencia. Calidad de Vida.

\section{ABSTRACT}

"FINASTERIDE: TEN YEARS OF CLINICAL USE. SISTEMATIC REVISION OF BIBLIOGHRAPHY"

INTRODUCTION: After ten years of clinical use of Finasteride in patientes with BPH, we carried out a systematic review of the literature including the assessment of their quality and grading the level of evidence for clinical recommendations.

METHODS: Using Medline, Embase, Healthstar and Cochrane Library from 1990 until 2002, we select all the studies refering patients between 50 to 85 with symptoms of BPH, metrics of flow, prostatic volume, postmictional residue, detrusor pressure, adverse effects, cost-efecctiveness and quality of life.

RESULTS: We found out 135 references, of which 36 accomplish the inclusion criteria. Of those, 3 have got level I of evidence, 12 level II, 6 level III and 10 level IV. Three are economic studies and two evaluate the quality of life.

DISCUSSION: With a high level of evidence and after ten years of clinical use, Finasteride shows its effectiveness in reducing the symptoms of patients with prostate bigger than $40 \mathrm{ml}$ and/or PSA of more than $1.4 \mathrm{ngr} / \mathrm{ml}$, with scarce adverse effects with a clear improvement of quality of life. Therefore, it is recommended (grade A) for clinical use.

CONCLUSIONS: Finasteride, through prostate volume reduction, modify natural evolution of BPH, decreasing the risk of acute urine retention and surgery.

KEY WORDS: HBP. Finasteride. Evidence based Medicina. Quality of life. 
El objetivo fundamental del tratamiento del paciente con hiperplasia benigna de próstata (H.B.P) es la mejora de su sintomatología y calidad de vida.

La sintomatología puede ser producida por incremento del tamaño prostático y/o aumento de la actividad alfa-adrenérgica, con obstrucción del tramo urinario inferior.

Los estudios epidemiológicos y de evolución natural de los pacientes con sintomatología del aparato urinario inferior secundarios a H.P.B., nos indican que la edad, el nivel sintomático, el volumen prostático y el valor de flujo urinario máximo son indicadores del riesgo que tiene el paciente de necesitar una intervención quirúrgica o de sufrir retención aguda de orina (R.A.O.). Aquellos pacientes con más de un factor de riesgo tendrán una probabilidad mayor de R.A.O.

Los tratamientos disponibles deben cumplir los criterios siguientes para que sean considerados una opción aceptable:

- Estudios de Eficacia y Seguridad en ensayos clínicos aleatorizados, controlados con placebo y con un seguimiento mínimo de 1 año de duración.

- Que sean fármacos que mejoren la sintomatología del paciente y/o prevengan las complicaciones.

- Que tengan una morbilidad y mortalidad mínimas, comparándose con el tratamiento estándar o con la resección transuretral de próstata (R.T.U.) y que no interfieran con la calidad de vida del paciente, y por último.

- Que tengan estudios a largo plazo manteniendo la demostración de eficacia y seguridad, que se compare con tratamientos ya existentes y que se realicen estudios costeefectividad con los tratamientos nuevos y los ya existentes.

Dentro de las opciones de tratamiento son consideradas de primera línea por el último consenso internacional de la Organización Mundial de la Salud: los antagonistas de los receptores alfa adrenérgicos cuyas moléculas más importantes son alfuzosina, doxazosina, terazosina y tamsulosina y los inhibidores de la 5-alfa-reductasa cuyas moléculas más importantes son finasterida y dutasterida.
Las otras opciones de tratamiento tal como "esperar y ver" se debería utilizar en pacientes con síntomas leves y poca afectación de la calidad de vida. El tratamiento quirúrgico es la última opción y la más agresiva, con una morbilidad (hematuria, estenosis de uretra, infección urinaria, orquiepididimitis, etc.) que se sitúa entre un $15-20 \%$. Es una opción a plantear a los pacientes con síntomas moderados y severos e importante afectación de la calidad de vida, que no aceptan o no responden al tratamiento farmacológico.

Un último factor a considerar es la existencia de comorbilidad asociada a la H.P.B. siendo bastante frecuente la asociación entre cardiopatía isquémica, hipertensión arterial y H.P.B.

Ha transcurrido una década desde la introducción clínica de Finasterida, en el tratamiento de la H.P.B. Por ello nos planteamos revisar la literatura, con los criterios antes señalados, para valorar la situación actual del fármaco.

\section{MATERIAL Y MÉTODO}

Se establecen unos criterios de inclusión que deben de cumplir aquellos trabajos que van a ser finalmente revisados. $\mathrm{El}$ análisis se realiza sobre los estudios cuyo diseño se corresponden con ensayos clínicos controlados y aleatorizados, meta-análisis, estudios mixtos de seguimiento con intervención (extensiones abiertas de los ensayos clínicos), estudios de calidad de vida relacionada con la salud, y estudios fármaco-económicos.

Los trabajos analizados incluyen pacientes con edades comprendidas entre 50 y 85 años con síntomas de aparato urinario inferior sugerentes de HBP, mediciones del flujo urinario máximo (FUM) comprendidas entre $5 \mathrm{ml}$ y $15 \mathrm{ml} / \mathrm{sg}$, cuya presión del detrusor (músculo vesical) a flujo máximo fuera mayor de $50 \mathrm{~cm}$ de agua, presentando volúmenes de orina residual mayores de $150 \mathrm{ml} \mathrm{y}$ agrandamiento prostático comprobado por palpación digital (DRE), ecografía, Tomografía Axial Computarizada o Resonancia Nuclear Magnética. El tipo de intervención consiste en el tratamiento con finasterida comparado con placebo o bien finasterida comparado con alfabloqueantes $o$ extractos de plantas solos o en combinación.

Se excluyeron aquellos estudios cuyos pacientes incluian sujetos con evidencias de infección urinaria activa, prostatitis crónica, vejiga neurógena o carcinoma prostático. 
Se analizaron los siguientes resultados:

- Síntomas de aparato urinario inferior evaluados por cuestionarios como el International Prostatic Symptom Score (IPSS), American Urological Association Symptom Index (AUASI) y Boyarski.

- Calidad de vida con cuestionarios como el BPH Impact Index (BII).

- Medida Volumen prostático (VP).

- Flujo urinario máximo (FUM).

- Influencia sobre la historia natural de la enfermedad:

- Incidencia de retención urinaria aguda (AUR).

- Incidencia de tratamiento quirúrgico.

- Efectos Adversos (EA) relacionados con el medicamento: disfunción en la esfera sexual.

- Evaluación de coste-efectividad.

La estrategia de localización de los trabajos científicos se centra en la utilización de las bases de datos informáticas disponibles en Medline, Embase, Healthstar y la Cochrane Library desde 1990 hasta junio de 2002. Para la localización de los trabajos publicados se utilizaron términos en lenguaje controlado con los diferentes Mesh: "finasteride", "benign prostatic hyperplasia", "prostate" y los distintos subheadings: cost, economics, quality, adverse events; combinados y relacionados mediante los diferentes operadores boléanos. La utilización en la estrategia de búsqueda de filtros metodológicos mejoró la localización de aquellos trabajos con un nivel de evidencia óptimo: meta-análisis, revisiones sistemáticas y ensayos clínicos. Quedan recogidos por tanto, aquellos ensayos clínicos aleatorizados de muestra grande ( $>500$ pacientes), ensayos clínicos aleatorizados de muestra pequeña $(<500$ pacientes), meta-análisis, estudios mixtos de seguimiento con intervención (extensiones abiertas de los ensayos clínicos), estudios de calidad de vida relacionada con la salud, y estudios fármaco-económicos.

Dos revisores independientes seleccionaron los estudios para su inclusión valorando el nivel de calidad de cada uno, las diferencias fueron resueltas tras discusión. Se analizan y clasifican los artículos según la escala de clasificación del nivel de evidencia científica, para así establecer de forma jerárquica diferentes niveles de la evidencia en función del rigor científico del diseño del estudio.
En nuestro análisis partimos de los criterios de la AATM (Agéncia d'Avaluació de Técnología Médica) ${ }^{1}$ (Tabla I).

Posteriormente a partir del análisis y de la evaluación de la evidencia recogida se formulan las recomendaciones, según la graduación de la calidad de la evidencia del estudio. Se clasifican con el grado A (Buena) cuando es posible formular recomendaciones favorables a su utilización, correspondiéndose en la escala de calidad de la evidencia, con las conclusiones derivadas de meta-análisis y ensayos clínicos bien diseñados y de muestra grande y pequeña. Como grado $\mathrm{B}$ (Aceptable evidencia científica) cuando las recomendaciones no son concluyentes como es el caso de los ensayos clínicos controlados no aleatorizados, estudios de cohortes y casos control. Con el grado C (Baja), cuando la evidencia científica es insuficiente y la adopción de la tecnología debe basarse en otros criterios, incluye los estudios descriptivos, series clínicas no controladas, comités de expertos y anécdotas (Tabla II).

TABLA I

NIVELES DE EVIDENCIA SEGÚN EL DISEÑO DEL ESTUDIO

\begin{tabular}{|c|c|c|}
\hline Nivel & Tipo diseño & Condiciones \\
\hline I & $\begin{array}{l}\text { Meta-análisis de } \\
\text { ensayos controlados } \\
\text { y aleatorizados }\end{array}$ & $\begin{array}{l}\text { No heterogeneidad } \\
\text { Diferentes técnicas de análisis } \\
\text { Metarregresión } \\
\text { Mega-análisis } \\
\text { Calidad de los estudios }\end{array}$ \\
\hline II & $\begin{array}{l}\text { Ensayo controlado } \\
\text { y aleatorizado } \\
\text { de muestra grande }\end{array}$ & $\begin{array}{l}\text { Evaluación del poder estadístico } \\
\text { Multicéntrico } \\
\text { Calidad del estudio }\end{array}$ \\
\hline III & $\begin{array}{l}\text { Ensayo controlado } \\
\text { y aleatorizado } \\
\text { de muestra pequeña }\end{array}$ & $\begin{array}{l}\text { Evaluación del poder estadístico } \\
\text { Calidad del estudio }\end{array}$ \\
\hline IV & $\begin{array}{l}\text { Ensayo prospectivo } \\
\text { controlado no } \\
\text { aleatorizado }\end{array}$ & $\begin{array}{l}\text { Controles coincidentes } \\
\text { en el tiempo } \\
\text { Multicéntrico } \\
\text { Calidad de estudio }\end{array}$ \\
\hline $\mathrm{V}$ & $\begin{array}{l}\text { Ensayo prospectivo } \\
\text { controlado no } \\
\text { aleatorizado }\end{array}$ & $\begin{array}{l}\text { Controles históricos } \\
\text { Calidad del estudio } \\
\text { Multicéntrico }\end{array}$ \\
\hline VI & $\begin{array}{l}\text { Estudios de cohorte } \\
\text { Calidad del estudio }\end{array}$ & Apareamiento \\
\hline VII & $\begin{array}{l}\text { Estudios de casos controles } \\
\text { Calidad del estudio }\end{array}$ & Multicéntrico \\
\hline VIII & $\begin{array}{l}\text { Series clinicas no controladas } \\
\text { Estudios descriptivos } \\
\text { Comités de expertos }\end{array}$ & Multicéntrico \\
\hline IX & Anécdotas o casos únicos & \\
\hline
\end{tabular}

AATM (Agéncia d’Avaluació de Tecnología Médica) 
TABLA II

NIVEL DE CALIDAD DE LA EVIDENCIA Y GRADO DE RECOMENDACIÓN

\begin{tabular}{||l|l|l|}
\hline $\begin{array}{l}\text { Nivel de calidad de la } \\
\text { evidencia científica }\end{array}$ & $\begin{array}{c}\text { Grado de } \\
\text { recomendación }\end{array}$ & Recomendación \\
\hline $\begin{array}{l}\text { I: Ensayos aleatorizados con una } \\
\text { muestra grande y resultados bien } \\
\text { definidos (y riesgo bajo de error } \\
\text { estadístico alfa y beta) }\end{array}$ & $\begin{array}{c}\text { A } \\
\text { Buena }\end{array}$ & $\begin{array}{l}\text { Existe adecuada evidencia para } \\
\text { recomendar o desaconsejar la } \\
\text { adopción de la tecnología. }\end{array}$ \\
$\begin{array}{l}\text { II: Ensayos aleatorizados con una } \\
\text { muestra pequeña (y un riesgo } \\
\text { moderado a alto de error estadístico } \\
\text { tipo alfa y beta) }\end{array}$ & B \\
$\begin{array}{l}\text { III: Estudios no aleatorizados, } \\
\text { controles concurrentes en el tiempo }\end{array}$ & Aceptable \\
$\begin{array}{l}\text { IV: Estudios no aleatorizados, } \\
\text { controles históricos }\end{array}$ & C & $\begin{array}{l}\text { Existe cierta evidencia científica } \\
\text { para recomendar no desaconsejar } \\
\text { la adopción de la tecnología. }\end{array}$ \\
$\begin{array}{l}\text { V: Estudios no controlados, series } \\
\text { clinicas }\end{array}$ & Baja & $\begin{array}{l}\text { Existe insuficiente evidencia } \\
\text { científica para recomendar o } \\
\text { desaconsejar la adopción de la } \\
\text { tecnología. }\end{array}$ \\
\hline
\end{tabular}

La localización de los estudios procedentes de las bases de datos empleadas proporcionó un total de 137 referencias de las cuales fueron seleccionadas por cumplir los criterios de inclusión un total de 36, una vez descartados previamente aquellos trabajos cuyos pacientes estaban incluidos en estudios publicados anteriormente (Tabla III). Los trabajos recogidos en la Tabla IV resumen las características más relevantes de los estudios seleccionados, analizando la calidad de la evidencia que aportan, y en el caso de los ensayos clínicos el nivel de calidad del diseño de los mismos. Por su claridad, rapidez y sencillez hemos adoptado la propuesta por Jaddad $^{2}$ (Tabla V) que es además una de las de mayor aceptación.

\section{COMENTARIOS}

De los 36 estudios analizados, 3 tienen el máximo nivel de evidencia científica (nivel I) 12 son nivel II, 6 nivel III y 10 nivel IV.

Los 5 restantes corresponden a estudios de evaluación económica (tres de ellos) y dos valoran la satisfacción del paciente y/o calidad de vida.

21.396 pacientes han sido evaluados en estos 36 estudios.

Si consideramos los tres meta-análisis realizados (nivel I de evidencia) todos ellos establecen la respuesta a Finasterida con más de un año de seguimiento. En el llevado a cabo por Boyle et al., en $1996^{16}$, se observa que el volumen prostático constituye un parámetro muy importante para

\section{TABLA III}

DISEÑO DE LOS ESTUDIOS RECOGIDOS EN LA REVISIÓN SISTEMÁTICA

\begin{tabular}{|l|c|c|c|c|}
\hline & Meta-análisis & $\begin{array}{c}\text { Ensayos } \\
\text { clínicos }\end{array}$ & $\begin{array}{c}\text { Estudios mixtos de seguimiento } \\
\text { con intervención }\end{array}$ & Otros \\
\hline Efectividad y seguridad frente placebo & 3 & 14 & 9 & 1 \\
\hline Eficacia Finasterida vs otros medicamentos & 4 & & 1 \\
\hline CVRS & & 1 & 3 \\
\hline Farmacoeconómicos & & & & 1 \\
\hline
\end{tabular}


TABLA IV

CARACTERIZACIÓN DE LOS ESTUDIOS REVISADOS

\begin{tabular}{|c|c|c|c|c|c|c|c|}
\hline Autor & Diseño & Población & Intervención & Resultados & Conclusiones & $\begin{array}{l}\text { Nivel de } \\
\text { evidencia }\end{array}$ & $\begin{array}{c}\text { Calidad } \\
\text { ECC }\end{array}$ \\
\hline $\begin{array}{l}\text { Beisland }^{3} \\
1992 \\
\text { Scandinavian } \\
\text { Finasteride } \\
\text { Study } \\
\text { Group }\end{array}$ & $\begin{array}{l}\text { Doble ciego } \\
\text { controlado } \\
\text { Multicéntrico }\end{array}$ & $\begin{array}{l}182 \text { pacientes } \\
\text { Aleatorizado }\end{array}$ & $\begin{array}{l}24 \text { semanas } 5 \mathrm{mg} \\
\text { finasteride/placebo } \\
+ \\
\text { extensión de } 12 \\
\text { meses de finasterida }\end{array}$ & $\begin{array}{l}\text { Boyarsky }-2 \text { ptos del } \\
\text { score } \\
\text { VP(<20\%) }-22,5 \% \\
\text { FUM + 1,6 ml/seg } \\
\text { EA impotencia 6,6\% }\end{array}$ & $\begin{array}{l}\text { No disminución } \\
\text { significativa de los } \\
\text { sintomas } \\
\text { Disminución del VP, } \\
\text { incremento del flujo } \\
\text { urinario } \\
\text { Buena tolerancia }\end{array}$ & III & 3 \\
\hline Kirby ${ }^{4} 1992$ & $\begin{array}{l}\text { Doble ciego } \\
\text { controlado } \\
\text { aleatorizado }\end{array}$ & 69 pacientes & $\begin{array}{l}5,10 \mathrm{mg} \\
\text { finasterida/placebo } \\
3 \text { meses } \\
\text { extensión a } 12 \text { meses }\end{array}$ & $\begin{array}{l}\text { Boyarsky -4 puntos F } \\
5 \mathrm{mg} \\
\text { VP }-14 \% \\
\text { FUM }+3,3 \mathrm{ml} / \mathrm{seg} \\
\text { EA } 1 \text { casos de } \\
\text { disfunción eréctil }\end{array}$ & $\begin{array}{l}\text { Disminución de los } \\
\text { sintomas } \\
\text { Disminución del VP, } \\
\text { aumento del flujo } \\
\text { urinario máximo } \\
\text { Baja toxicidad del } \\
\text { fármaco }\end{array}$ & III & 3 \\
\hline $\begin{array}{l}\text { Gormley }{ }^{5} \\
1992 \\
\text { Finasterida } \\
\text { Study group }\end{array}$ & $\begin{array}{l}\text { Doble ciego } \\
\text { controlado } \\
\text { Multicéntrico } \\
\text { Aleatorizado }\end{array}$ & 895 pacientes & $\begin{array}{l}1 \text { y } 5 \mathrm{mg} \\
\text { finasterida/placebo } \\
12 \text { meses }\end{array}$ & $\begin{array}{l}\text { Boyarsky - } 21 \% \\
\text { FUM } \\
+1,6 \mathrm{ml} / \mathrm{sg}(22 \%) \\
\text { VP - } 19 \% \\
\text { EA 0,5\% de los } \\
\text { casos }\end{array}$ & $\begin{array}{l}5 \mathrm{mg} / \mathrm{d} \text { produce una } \\
\text { disminución } \\
\text { significativa de los } \\
\text { sintomas } \\
\text { obstructivos, flujo } \\
\text { urinario y } \\
\text { disminución del } \\
\text { volumen prostático. } \\
\text { Mayor incidencia de } \\
\text { disfunción sexual en } \\
\text { grupo Finasterida }\end{array}$ & II & 4 \\
\hline $\begin{array}{l}\text { Stoner }^{6} \\
1992\end{array}$ & $\begin{array}{l}\text { Doble ciego } \\
\text { controlado } \\
\text { aleatorizado }\end{array}$ & $\begin{array}{l}86 \text { pacientes } \\
\text { estudio } 1 \\
104 \text { en el } \\
\text { estudio } 2\end{array}$ & $\begin{array}{l}\text { De } 5 \text { a } 80 \mathrm{mg} \\
\text { finasterida } \\
12 \text { semanas tto y } 12 \\
\text { semanas de lavado } \\
\text { estudio } 2: 0,2-40 \mathrm{mg} \\
\text { finasterida } 6 \text { meses }\end{array}$ & $\begin{array}{l}\text { Boyarsky mejoria en } \\
\text { el grupo } 2 \\
\text { VP - } 28 \% \text { en } 24 \\
\text { semanas } \\
\text { FUM }+3,7 \mathrm{ml} / \mathrm{s} \\
\text { Seguridad: efectos } 2^{\text {a }} \\
\text { no atribuibles }\end{array}$ & $\begin{array}{l}\text { Mejoria de los } \\
\text { sintomas } \\
\text { obstructivos. Gradual } \\
\text { disminución del VP. } \\
\text { Incremento del FUM } \\
\text { Eficacia y seguridad } \\
\text { del finasterida hasta } \\
80 \mathrm{mg} / \mathrm{d}\end{array}$ & III & 3 \\
\hline $\begin{array}{l}\text { Kirby }^{7} \\
1993\end{array}$ & $\begin{array}{l}\text { Estudio mixto } \\
\text { de } \\
\text { seguimiento } \\
\text { con } \\
\text { intervención }\end{array}$ & 69 pacientes & $\begin{array}{l}5,10 \mathrm{mg} \\
\text { finasterida/placebo } \\
3 \text { meses } \\
\text { extensión a } 3 \text { años } 10 \\
\text { pacientes }\end{array}$ & $\begin{array}{l}\text { Boyarsky }-5,4 \% \text { puntos } \\
\text { VP }-13,4 \% \\
\text { FUM }+5,1 \mathrm{ml} / \mathrm{seg} \\
\text { Presión vesical }-28 \mathrm{~cm} \\
\text { H2O }\end{array}$ & $\begin{array}{l}\text { El máximo efecto del } \\
\text { finasterida sobre la } \\
\text { mejoría de la } \\
\text { obstrucción urinaria } \\
\text { no se observa hasta } \\
\text { pasado un año de } \\
\text { tratamiento }\end{array}$ & IV & \\
\hline $\begin{array}{l}\text { Ekman }^{8} \\
1993\end{array}$ & $\begin{array}{l}\text { Doble ciego } \\
\text { controlado } \\
\text { aleatorizado }\end{array}$ & $\begin{array}{l}1645 \\
\text { pacientes }\end{array}$ & $\begin{array}{l}1,5 \mathrm{mg} \text { de } \\
\text { finasterida/placebo } \\
12 \text { meses }\end{array}$ & $\begin{array}{l}\text { IPSS -3,9\% puntos con } \\
5 \mathrm{mg} \\
\mathrm{VP}-22 \% \\
\mathrm{FUM}+1,7 \mathrm{ml} / \mathrm{s} \\
\text { Impotencia y } \\
\text { disminución } \\
\text { líbido: } 3,4 \%\end{array}$ & $\begin{array}{l}\text { Pacientes con } \\
\text { sintomatologia leve y } \\
\text { moderada son el } \\
\text { grupo que obtiene un } \\
\text { mayor beneficio. }\end{array}$ & II & 4 \\
\hline $\begin{array}{l}\text { Stoner } 9 \\
1994 \\
\text { Finasteride } \\
\text { Study } \\
\text { Group }\end{array}$ & $\begin{array}{l}\text { Estudios } \\
\text { mixtos de } \\
\text { seguimiento } \\
\text { con } \\
\text { intervención }\end{array}$ & 874 pacientes & $\begin{array}{l}1 \text { y } 5 \mathrm{mg} \text { finasterida } \\
12 \text { meses } \\
\text { estudio de extensión } \\
5 \mathrm{mg} 24 \text { meses }\end{array}$ & $\begin{array}{l}\text { Boyarsky - } 3,5 \text { puntos } \\
\text { VP }-25 \% \\
\text { FUM }+2,3 \mathrm{ml} / \text { seg en } \\
\text { internacional y } 2 \mathrm{ml} / \mathrm{s} \\
\text { en norteamericano } \\
\mathrm{AE} \text { : Impotencia } 4,8 \\
\text { y disminución líbido } \\
4,2\end{array}$ & $\begin{array}{l}\text { La reducción de los } \\
\text { sintomas, reducción } \\
\text { del VP y FUM se } \\
\text { mantienen durante los } \\
24 \text { meses } \\
\text { No aumentan los } \\
\text { efectos adversos con } \\
\text { la extensión del } \\
\text { tratamiento }\end{array}$ & IV & \\
\hline
\end{tabular}


TABLA IV (Continuación)

CARACTERIZACIÓN DE LOS ESTUDIOS REVISADOS

\begin{tabular}{|c|c|c|c|c|c|c|c|}
\hline Autor & Diseño & Población & Intervención & Resultados & Conclusiones & $\begin{array}{l}\text { Nivel de } \\
\text { evidencia }\end{array}$ & $\begin{array}{l}\text { Calidad } \\
\text { ECC }\end{array}$ \\
\hline $\begin{array}{l}\text { Andersen }{ }^{10} \\
1995 \\
\text { Scandinavia } \\
\text { n BHP } \\
\text { Study } \\
\text { Group }\end{array}$ & $\begin{array}{l}\text { Doble ciego } \\
\text { controlado } \\
\text { Multicéntrico } \\
\text { aleatorizado }\end{array}$ & 707 pacientes & $\begin{array}{l}5 \mathrm{mg} \text { finasterida } 24 \\
\text { meses vs Placebo }\end{array}$ & $\begin{array}{l}\text { Boyarsky - } 2 \text { puntos } \\
\text { FUM }>1,5 \mathrm{ml} / \mathrm{seg} \\
\text { VP }<19 \% \\
\text { EA esfera sexual 19\% } \\
\text { grupo F vs } 10 \% \mathrm{P}\end{array}$ & $\begin{array}{l}\text { Mejoria significativa } \\
\text { de sintomas, FUM y } \\
\text { disminución volumen } \\
\text { que se mantienen } 2 \\
\text { años. Puede revertir } \\
\text { la historia natural }\end{array}$ & II & 4 \\
\hline $\begin{array}{l}\text { Byrnes }^{11} \\
1995\end{array}$ & $\begin{array}{l}\text { Doble ciego } \\
\text { Controlado } \\
\text { Multicéntrico } \\
\text { aleatorizado }\end{array}$ & $\begin{array}{l}2342 \\
\text { pacientes }\end{array}$ & $\begin{array}{l}5 \mathrm{mg} \text { finasterida vs } \\
\text { Placebo } 12 \text { meses }\end{array}$ & $\begin{array}{l}\text { AUASI - } 4,8 \text { puntos } \\
\text { BII: - } 1,2 \text { puntos } \\
\text { EA impotencia } 5,6 \text { y } \\
\text { 2,9\% de disminución } \\
\text { de la líbido }\end{array}$ & $\begin{array}{l}\text { Finasterida mejora la } \\
\text { calidad de vida en } \\
\text { pacientes con } \\
\text { sintomatología } \\
\text { moderada y severa }\end{array}$ & II & 3 \\
\hline $\begin{array}{l}\text { Geller }{ }^{12} \\
1995 \\
\text { Finasteride } \\
\text { Study } \\
\text { Group }\end{array}$ & $\begin{array}{l}\text { Estudios } \\
\text { mixtos de } \\
\text { seguimiento } \\
\text { con } \\
\text { intervención }\end{array}$ & 55 pacientes & $\begin{array}{l}5 \text { años } 5 \mathrm{mg} \\
20 \text { pacientes dosis } \\
\text { variables } 6 \text { meses } \\
\text { posteriormente } 5 \mathrm{mg} \\
4 \text { años }\end{array}$ & $\begin{array}{l}\text { Boyarsky }-2,5 \text { puntos } \\
\text { VP }-24 \% \\
\text { FUM + } 1,9 \mathrm{ml} / \mathrm{seg} \\
\mathrm{AE} 3 \text { casos de } \\
\text { impotencia reversible } \\
\text { tras abandono }\end{array}$ & $\begin{array}{l}\text { El Finasterida } \\
\text { modifica la evolución } \\
\text { de la HBP reduciendo } \\
\text { el tamaño prostático y } \\
\text { mejorando los } \\
\text { sintomas del paciente }\end{array}$ & IV & \\
\hline $\begin{array}{l}\text { Tammella }^{13} \\
1995\end{array}$ & $\begin{array}{l}\text { Estudio mixto } \\
\text { de } \\
\text { seguimiento } \\
\text { con } \\
\text { intervención }\end{array}$ & 36 pacientes & $\begin{array}{l}5 \text { mg finasterida } \\
6 \text { meses vs placebo } \\
+ \text { extensión de } 4,5 \\
\text { años } \\
\text { todos }\end{array}$ & $\begin{array}{l}\text { VP - } 21 \text { cc } \\
\text { FUM sin cambios } \\
\text { Pres Detrusor a flujo } \\
\text { máximo -70A77 ml } \\
\text { H2O } \\
5 \text { pacientes precisaron } \\
\text { cirugia }\end{array}$ & $\begin{array}{l}\text { El efecto del } \\
\text { finasterida se } \\
\text { incrementa a largo } \\
\text { plazo } \\
\text { Disminuye la } \\
\text { obstrucción de salida } \\
\text { y por ello mejora los } \\
\text { sintomas urinarios }\end{array}$ & IV & \\
\hline $\begin{array}{l}\text { Moore }^{14} \\
1995\end{array}$ & $\begin{array}{l}\text { Estudio mixto } \\
\text { De } \\
\text { seguimiento } \\
\text { con } \\
\text { intervención }\end{array}$ & 70 pacientes & $\begin{array}{l}\text { Estudio } 1: 10 \mathrm{mg} \\
\text { Finasterida } 1 \text { año }+4 \\
\text { años } 5 \mathrm{mg} \\
\text { Estudio } 2: 5 \text { años } 5 \\
\text { mg finasterida }\end{array}$ & $\begin{array}{l}\text { Boyarsky -1 punto } \\
\text { VP - } 30 \% \\
\text { FUM + 1,5 ml/s } \\
\text { EA: eyaculación } \\
5,7 \% \\
\text { libido e impotencia: } \\
4,3 \% \\
\text { RTU 5,9\% }\end{array}$ & $\begin{array}{l}\text { El tratamiento con } \\
\text { finasterida a largo } \\
\text { plazo inhibe la } \\
\text { progresión de la } \\
\text { enfermedad }\end{array}$ & IV & \\
\hline $\begin{array}{l}\text { Nickel }^{15} \\
1996 \\
\text { PROSPEC } \\
\text { T Study }\end{array}$ & $\begin{array}{l}\text { Doble ciego } \\
\text { controlado } \\
\text { Multicéntrico } \\
\text { aleatorizado }\end{array}$ & 613 pacientes & $\begin{array}{l}5 \mathrm{mg} \\
\text { finasterida/placebo } \\
2 \text { años }\end{array}$ & $\begin{array}{l}\text { IPSS - } 2,1 \text { puntos } \\
\text { VP - } 21 \% \\
\text { FUM + } 1,4 \mathrm{ml} / \mathrm{s} \\
\text { EA alteración } \\
\text { eyaculación }+6 \% \\
\text { Impotencia }+8,5 \% \\
\text { Retención urinaria } \\
6,1 \% \text { vs } 10,2\end{array}$ & $\begin{array}{l}2 / 3 \text { de los pacientes } \\
\text { mejoran con } \\
\text { Finasterida y el efecto } \\
\text { se mantiene durante } \\
\text { al menos } 2 \text { años } \\
\text { Disminución } \\
\text { significativa de } \\
\text { episodios de } \\
\text { retención urinaria }\end{array}$ & II & 5 \\
\hline $\begin{array}{l}\text { Boyle }^{16} \\
1996\end{array}$ & Meta-análisis & $\begin{array}{l}2061 \\
\text { pacientes } \\
6 \mathrm{ECC}\end{array}$ & $\begin{array}{l}5 \mathrm{mg} \text { de finasterida > } \\
\text { de } 1 \text { año }\end{array}$ & $\begin{array}{l}\text { En pacientes con } \\
\text { VP >40 cc: } \\
\text { IPSS mejora } 2,8 \text { ptos } \\
\text { FUM mejora } 1,84 \\
\mathrm{ml} / \mathrm{s}\end{array}$ & $\begin{array}{l}\text { Finasterida es más } \\
\text { efectivo en pacientes } \\
\text { con próstatas de gran } \\
\text { tamaño VP > } 40 \text { cc }\end{array}$ & I & \\
\hline $\begin{array}{l}\text { Carraro }^{17} \\
1996\end{array}$ & $\begin{array}{l}\text { Doble ciego } \\
\text { controlado } \\
\text { Multicéntrico } \\
\text { aleatorizado }\end{array}$ & $\begin{array}{l}1098 \\
\text { pacientes }\end{array}$ & $\begin{array}{l}\text { Permixon (P) } 320 \\
\text { mg/finasterida }(\mathrm{F}) \\
5 \mathrm{mg} \\
6 \text { meses }\end{array}$ & $\begin{array}{l}\text { IPSS - } 37 \% \text { P vs - } 39 \\
\% \text { F n.s } \\
\text { QALY +38\% P vs } \\
+41 \mathrm{~F} \text { n.s } \\
\text { FUM + } 25 \text { P vs }+30 \% \mathrm{~F} \\
\text { VP - } 6 \% \text { P vs }-18 \% \mathrm{~F} \\
\text { Sexual function Score } \\
-6 \% \text { P vs }+9 \mathrm{~F}\end{array}$ & $\begin{array}{l}\text { Ambos tratamientos } \\
\text { mejoran los sintomas } \\
\text { en } 2 / 3 \text { de los } \\
\text { pacientes. Finasterida } \\
\text { tiene mayor efecto } \\
\text { sobre parámetros } \\
\text { andrógenos } \\
\text { dependientes }\end{array}$ & II & 4 \\
\hline
\end{tabular}


TABLA IV (Continuación)

CARACTERIZACIÓN DE LOS ESTUDIOS REVISADOS

\begin{tabular}{|c|c|c|c|c|c|c|c|}
\hline Autor & Diseño & Población & Intervención & Resultados & Conclusiones & $\begin{array}{l}\text { Nivel de } \\
\text { evidencia }\end{array}$ & $\begin{array}{l}\text { Calidad } \\
\text { ECC }\end{array}$ \\
\hline $\begin{array}{l}\text { Lepor }^{18} \\
1996\end{array}$ & $\begin{array}{l}\text { Controlado } \\
\text { Multicéntrico } \\
\text { aleatorizado } \\
\text { Mono ciego }\end{array}$ & $\begin{array}{l}1229 \\
\text { pacientes }\end{array}$ & $\begin{array}{l}52 \text { semanas } \\
\text { Finasterida } \\
5 \mathrm{mg} / \text { Terazosina } \\
10 \mathrm{mg} / \mathrm{F}+\mathrm{T} \text { vs Placebo }\end{array}$ & $\begin{array}{l}\text { AUASS -2,6P, } 3,2 \mathrm{~F} \text {, } \\
6,1 \mathrm{~T}, 6,2 \mathrm{~F}+\mathrm{T} \\
\text { FUM +1,4 P, 1,6 F, } \\
2,7 \mathrm{~T}, 3,2 \mathrm{~F}+\mathrm{T} \\
\text { VP -6,1 cc F, +0,5 cc } \\
\mathrm{T},-7 \mathrm{cc} \mathrm{F}+\mathrm{T} \\
\text { Eventos adversos } \\
1,6 \% \mathrm{P}, 4,8 \mathrm{~F}, 5,7 \mathrm{~T}, \\
7,7 \mathrm{~T}+\mathrm{F}\end{array}$ & $\begin{array}{l}\text { Mayor eficacia de la } \\
\text { terazosina en mejoria } \\
\text { de la sintomatologia } \\
\text { obstructiva y flujo } \\
\text { urinario. La } \\
\text { combinación de } \\
\text { ambas no es mejor } \\
\text { que la terazosina sola }\end{array}$ & II & 2 \\
\hline $\begin{array}{l}\text { Martinez } \\
\text { Sarmiento }^{19} \\
1997 \\
\text { Finasteride } \\
\text { Study } \\
\text { Group }\end{array}$ & $\begin{array}{l}\text { Estudio mixto } \\
\text { de } \\
\text { seguimiento } \\
\text { con } \\
\text { intervención }\end{array}$ & 7 pacientes & $\begin{array}{l}\text { 1-5 mg de } \\
\text { finasterida/placebo } 12 \\
\text { meses } \\
\text { extensión abierta con } \\
\text { finasterida } 5 \text { mg hasta } \\
7 \text { años }\end{array}$ & $\begin{array}{l}\text { Boyarsky -6,2 puntos } \\
\text { FUM }+47,5 \%(4,2 \\
\mathrm{ml} / \mathrm{s}) \\
\text { VP - } 26 \% \\
\text { EA } 3 \text { casos }\end{array}$ & $\begin{array}{l}\text { La eficacia del } \\
\text { finasterida se } \\
\text { mantiene a los } 7 \text { años, } \\
\text { con un excelente } \\
\text { perfil de seguridad }\end{array}$ & IV & \\
\hline $\begin{array}{l}\text { Tenover }{ }^{20} \\
1997 \\
\text { Primary } \\
\text { Care Study } \\
\text { Group }\end{array}$ & $\begin{array}{l}\text { Doble ciego } \\
\text { controlado } \\
\text { Multicéntrico } \\
\text { aleatorizado }\end{array}$ & $\begin{array}{l}2112 \\
\text { pacientes }\end{array}$ & $\begin{array}{l}12 \text { meses } \\
\text { finasterida } \\
5 \mathrm{mg} / \text { placebo }\end{array}$ & $\begin{array}{l}\text { AUASI mejora } 1,2 \\
\text { puntos } \\
\text { BII mejora 0,4 puntos } \\
\text { EA en la esfera } \\
\text { sexual: impotencia } \\
8,1 \text { vs 3,8\% Py } \\
\text { alteración de la } \\
\text { eyaculación } 4 \text { vs } \\
\text { 0,9\% P } \\
\text { AUR 0,2\% } \\
\text { RTU 0,8\% }\end{array}$ & $\begin{array}{l}\text { La utilización de } \\
\text { finasterida mejora la } \\
\text { calidad de vida y la } \\
\text { sintomatologia con } \\
\text { una mayor incidencia } \\
\text { de efectos adversos } \\
\text { que en el } 2,2 \% \\
\text { produce un abandono } \\
\text { del tratamiento }\end{array}$ & II & 4 \\
\hline $\begin{array}{l}\text { Andersen }^{21} \\
1997\end{array}$ & Meta-análisis & $\begin{array}{l}4222 \\
\text { pacientes }\end{array}$ & $\begin{array}{l}2 \text { años finasterida } \\
5 \mathrm{mg} / \text { placebo }\end{array}$ & $\begin{array}{l}\text { AUR - Riesgo } \\
\text { Relativo del } 57 \% \\
\text { Cirugia - Riesgo } \\
\text { relativo del } 34 \%\end{array}$ & $\begin{array}{l}\text { El tratamiento } \\
\text { durante } 2 \text { años reduce } \\
\text { a la mitad la } \\
\text { frecuencia de AUR o } \\
\text { necesidad de } \\
\text { tratamiento } \\
\text { quirúrgico }\end{array}$ & I & \\
\hline $\begin{array}{l}\text { Kuo Hann } \\
\text { Chorng } 22 \\
1998\end{array}$ & $\begin{array}{l}\text { Estudio mixto } \\
\text { de } \\
\text { seguimiento } \\
\text { con } \\
\text { intervención }\end{array}$ & 190 pacientes & $\begin{array}{l}\text { Dibenyline } 10 \mathrm{mg} / \\
\text { Finasterida } 5 \mathrm{mg} \\
\mathrm{F}+\mathrm{D} \\
6 \text { meses }\end{array}$ & $\begin{array}{l}\text { IPSS en los tres } \\
\text { grupos } \\
\text { BII +79\% D, 70,4\% F } \\
\text { y } 83,1 \% \text { F+D } \\
\text { VP - } 24,3 \% \text { F y } 10,5 \% \\
\text { F+D } \\
\text { FUM: +1,4-1,8 ml/s } \\
\text { EA: impotencia } 16,9 \% \\
\text { D y F+D vs } 9,3 \% \text { F } \\
\text { RTU 1,9 F, 4,2 D y } \\
4,6 \% \text { F+D }\end{array}$ & $\begin{array}{l}\text { La combinación } \\
\text { Finasterida } \\
\text { Dibenyline produce } \\
\text { un débil efecto } \\
\text { sinérgico sin efecto } \\
\text { aditivo adverso }\end{array}$ & IV & \\
\hline $\begin{array}{l}\text { Ekman }^{23} \\
1998 \\
\text { Scandinavian } \\
\text { Finasteride } \\
\text { Study } \\
\text { Group }\end{array}$ & $\begin{array}{l}\text { Estudio mixto } \\
\text { de } \\
\text { seguimiento } \\
\text { con } \\
\text { intervención }\end{array}$ & 168 pacientes & $\begin{array}{l}6 \text { meses finasterida } \\
5 \mathrm{mg} \text { vs. Placebo } \\
\text { extensión } 6 \text { años }\end{array}$ & $\begin{array}{l}\text { IPSS + } 2,6 \\
\text { a } 3,4 \text { puntos (+30\%) } \\
\text { VP - } 28 \% \\
\text { FUM }+2,2 \mathrm{ml} / \mathrm{s}\end{array}$ & $\begin{array}{l}\text { Finasterida reduce el } \\
\text { VP del } 20-25 \% \text { en } 6 \\
\text { meses con mejoria de } \\
\text { los sintomas } \\
\text { obstructivos del } 30 \% \text { y } \\
\text { del FUM de } 2,2 \mathrm{ml} / \mathrm{s} \\
\text { mejorias que se } \\
\text { mantienen a los } 6 \text { años }\end{array}$ & IV & \\
\hline $\begin{array}{l}\text { Roehrborn }{ }^{24} \\
1998\end{array}$ & Meta-análisis & $\begin{array}{l}2741 \\
\text { pacientes } \\
6 \mathrm{ECC}\end{array}$ & $\begin{array}{l}>1 \text { año con } 5 \mathrm{mg} \\
\text { finasterida }\end{array}$ & $\begin{array}{l}\text { VP, FUM y IPSS } \\
\text { mejoran } \\
\text { significativamente en } \\
\text { pacientes con } \\
\text { VP }>40 \text { cc y tratados } \\
\text { con finasterida }\end{array}$ & $\begin{array}{l}\text { El VP al inicio es el } \\
\text { predictor del efecto } \\
\text { del finasterida siendo } \\
\text { este más efectivo en } \\
\text { próstatas de gran } \\
\text { tamaño }\end{array}$ & I & \\
\hline
\end{tabular}


TABLA IV (Continuación)

CARACTERIZACIÓN DE LOS ESTUDIOS REVISADOS

\begin{tabular}{|c|c|c|c|c|c|c|c|}
\hline Autor & Diseño & Población & Intervención & Resultados & Conclusiones & $\begin{array}{l}\text { Nivel de } \\
\text { evidencia }\end{array}$ & $\begin{array}{l}\text { Calidad } \\
\text { ECC }\end{array}$ \\
\hline $\begin{array}{l}\text { Marberger }^{25} \\
1998 \\
\text { PROWESS } \\
\text { Study } \\
\text { Group }\end{array}$ & $\begin{array}{l}\text { Doble ciego } \\
\text { controlado } \\
\text { multicéntrico } \\
\text { aleatorizado }\end{array}$ & $\begin{array}{l}3270 \\
\text { pacientes }\end{array}$ & $\begin{array}{l}2 \text { años finasterida } 5 \\
\mathrm{mg} / \mathrm{d} \text { vs placebo }\end{array}$ & $\begin{array}{l}\text { IPSS + } 1,7 \mathrm{ptos} \text { F vs P } \\
\text { FUM }+0,8 \mathrm{ml} / \mathrm{s} \\
\text { VP - } 15 \% \text { f vs } 9 \% \mathrm{P} \\
\text { Riesgo de AUR - } 57 \% \\
\text { y Cirugia - } 40 \%\end{array}$ & $\begin{array}{l}\text { Finasterida produce } \\
\text { una mejoria de los } \\
\text { sintomas y reduce } \\
\text { significativamente el } \\
\text { riesgo de AUR o } \\
\text { cirugía. El beneficio } \\
\text { es mayor cuanto } \\
\text { mayor es el tamaño } \\
\text { prostático }\end{array}$ & II & 5 \\
\hline $\begin{array}{l}\text { Debruyne }^{26} \\
1998 \\
\text { European } \\
\text { ALFIN } \\
\text { Study } \\
\text { Group }\end{array}$ & $\begin{array}{l}\text { Doble ciego } \\
\text { controlado } \\
\text { Multicéntrico } \\
\text { aleatorizado }\end{array}$ & $\begin{array}{l}1051 \\
\text { pacientes }\end{array}$ & $\begin{array}{l}\text { Alfuzosina } 5 \mathrm{mg} / \\
\text { Finasterida } 5 \mathrm{mg} / \mathrm{A}+\mathrm{F} \\
6 \text { meses }\end{array}$ & $\begin{array}{l}\text { IPSS -6,3 A, -6,1 } \\
\mathrm{A}+\mathrm{F},-5,2 \text { puntos } \\
\text { grupo } \mathrm{F} \\
\text { FUM } \\
\text { significativamente } \\
\text { mayor en } \mathrm{A} \text { y } \mathrm{A}+\mathrm{F}\end{array}$ & $\begin{array}{l}\text { Alfuzosina es más } \\
\text { efectiva que } \\
\text { finasterida sin } \\
\text { hallarse beneficio de } \\
\text { la combinación de } \\
\text { ambos }\end{array}$ & II & 4 \\
\hline $\begin{array}{l}\text { Abrams }^{27} \\
1999 \\
\text { Finasteride } \\
\text { Urodinamic } \\
\text { Study } \\
\text { Group }\end{array}$ & $\begin{array}{l}\text { Doble ciego } \\
\text { controlado } \\
\text { Multicéntrico } \\
\text { aleatorizado }\end{array}$ & 121 pacientes & $\begin{array}{l}12 \mathrm{meses} \text { finasterida } \\
5 \mathrm{mg} / \mathrm{ml} \text { vs placebo }\end{array}$ & $\begin{array}{l}\text { Global: } \\
\text { IPSS }-4,9 \text { ptos } \\
\text { FUM }+1,1 \mathrm{ml} / \mathrm{s} \\
\text { VP }-22,8 \% \\
\text { Ptes con VP }>40 \mathrm{ml} \text { : } \\
\text { FUM }+1,6 \mathrm{ml} / \mathrm{s} \\
\text { Presión detrusor a } \\
\text { flujo máximo }-14,5 \mathrm{~cm}\end{array}$ & $\begin{array}{l}\text { El tratamiento con } \\
\text { finasterida mejora los } \\
\text { parámetros } \\
\text { urodinámicos, } \\
\text { sobre todo en } \\
\text { pacientes con } \\
\text { próstatas grandes }\end{array}$ & III & 3 \\
\hline $\begin{array}{l}\text { Hudson28 } \\
1999 \\
\text { North } \\
\text { American } \\
\text { Finasterida } \\
\text { Study } \\
\text { Group }\end{array}$ & $\begin{array}{l}\text { Estudio mixto } \\
\text { de } \\
\text { seguimiento } \\
\text { con } \\
\text { intervención }\end{array}$ & 186 pacientes & $\begin{array}{l}12 \text { meses finasterida } \\
5 \mathrm{mg} \text { vs placebo }+ \\
\text { extensión abierta de } 4 \\
\text { años con finasterida } \\
5 \mathrm{mg}\end{array}$ & $\begin{array}{l}\text { AUASI -4,3 ptos } \\
\text { VP - } 22,7 \% \\
\text { FUM + 2,3 ml/s } \\
\text { AE: eyaculación } \\
2,7 \% \\
\text { Impotencia 9,6\%, } \\
\text { disminución de la } \\
\text { líbido 3,7\% } \\
\text { AUR - } 1,4 \% \\
\text { Cirugia - } 1 \%\end{array}$ & $\begin{array}{l}\text { Los pacientes tratados } \\
\text { con finasterida } 5 \mathrm{mg} \\
\text { durante } 5 \text { años } \\
\text { mantienen su mejoria } \\
\text { sintomática, } \\
\text { manteniendo la } \\
\text { disminución de VP } \\
\text { inicial y el } \\
\text { incremento del FUM }\end{array}$ & IV & \\
\hline $\begin{array}{l}\text { Schafer }^{29} \\
1999 \\
\text { Finasteride } \\
\text { Urodinamic } \\
\text { Study } \\
\text { Group }\end{array}$ & $\begin{array}{l}\text { Estudio mixto } \\
\text { de } \\
\text { seguimiento } \\
\text { con } \\
\text { intervención }\end{array}$ & 54 pacientes & $\begin{array}{l}12 \mathrm{meses} \text { finasterida } \\
5 \mathrm{mg} / \mathrm{ml} \text { o placebo y } \\
\text { finasterida } 12 \text { meses } \\
\text { más }\end{array}$ & $\begin{array}{l}\text { IPPS }-7,3 \text { puntos } \\
\text { FUM }+8 \mathrm{ml} / \mathrm{s}\end{array}$ & $\begin{array}{l}\text { Finasterida mejora } \\
\text { los parámetros } \\
\text { urodinámicos durante } \\
\text { un periodo de } 2 \text { años }\end{array}$ & IV & \\
\hline $\begin{array}{l}\text { Marks }^{30} \\
1999\end{array}$ & $\begin{array}{l}\text { Doble ciego } \\
\text { controlado } \\
\text { aleatorizado }\end{array}$ & 41 pacientes & $\begin{array}{l}6 \text { meses + extensión a } \\
2 \text { años }\end{array}$ & $\begin{array}{l}\text { IPSS + } 9 \text { ptos } \\
\text { VP - } 17,8 \% \\
\text { FUM - n.s. } \\
\text { Análisis } \\
\text { morfométrico - } 78 \% \\
\text { zona periférica del } \\
\text { epitelio }\end{array}$ & $\begin{array}{l}\text { El tratamiento a largo } \\
\text { plazo con finasterida } \\
\text { produce una } \\
\text { involución del } \\
\text { epitelio prostático } \\
\text { constituyendo este su } \\
\text { fundamental } \\
\text { mecanismo de acción }\end{array}$ & III & 3 \\
\hline $\begin{array}{l}\text { Sokeland }{ }^{31} \\
2000\end{array}$ & $\begin{array}{l}\text { Doble ciego } \\
\text { controlado } \\
\text { aleatorizado }\end{array}$ & 543 pacientes & $\begin{array}{l}48 \text { semanas } \\
2 \text { caps PRO 160/120/ } \\
5 \text { mg Finasterida }\end{array}$ & $\begin{array}{l}\text { IPSS similares } \\
\text { VP igual ambos } \\
\text { grupos } \\
\text { FUM no mejoria } \\
\text { significativa } \\
\text { Eventos adversos + n } \\
\text { grupo } \mathrm{F}\end{array}$ & $\begin{array}{l}\text { Los extractos de } \\
\text { Serenoa repens and } \\
\text { urtica dioica muestran } \\
\text { similar eficacia que el } \\
\text { Finasterida pero } \\
\text { mejor tolerancia }\end{array}$ & II & 4 \\
\hline
\end{tabular}


TABLA IV (Continuación)

CARACTERIZACIÓN DE LOS ESTUDIOS REVISADOS

\begin{tabular}{|c|c|c|c|c|c|c|c|}
\hline Autor & Diseño & Población & Intervención & Resultados & Conclusiones & $\begin{array}{l}\text { Nivel de } \\
\text { evidencia }\end{array}$ & $\begin{array}{l}\text { Calidad } \\
\text { ECC }\end{array}$ \\
\hline $\begin{array}{l}\text { Kaplan }^{32} \\
2001 \\
\text { PLESS } \\
\text { Study } \\
\text { Group }\end{array}$ & $\begin{array}{l}\text { Doble ciego } \\
\text { controlado } \\
\text { Multicéntrico } \\
\text { aleatorizado }\end{array}$ & $\begin{array}{l}3040 \\
\text { pacientes }\end{array}$ & $\begin{array}{l}4 \text { años } 5 \mathrm{mg} \text { de } \\
\text { finasterida vs. } \\
\text { placebo }\end{array}$ & $\begin{array}{l}\text { IPSS -2,2 puntos } \\
\text { RTU -5\% F vs 10\% } \\
\text { P(-51\% Riesgo } \\
\text { relativo) } \\
\text { AUR -del } 28-75 \% \\
\text { del riesgo relativo } \\
\text { AE: } \\
\text { Impotencia: -0,6\% } \\
\text { Líbido: -1\% } \\
\text { Eyaculación -1,3\% }\end{array}$ & $\begin{array}{l}\text { Finasterida tiene un } \\
\text { Efecto beneficioso } \\
\text { sobre los sintomas, } \\
\text { episodios de AUR y } \\
\text { necesidad de } \\
\text { tratamiento } \\
\text { quirúrgico, sobre todo } \\
\text { en los grupos de } \\
\text { mayor sintomatologia } \\
\text { de base y PSA mayor } \\
\text { de } 1,4 \text { ng/ml } \\
\text { No se detectaron } \\
\text { importantes } \\
\text { reacciones adversas al } \\
\text { medicamento }\end{array}$ & II & 3 \\
\hline $\begin{array}{l}\text { Girman }^{33} \\
1996 \\
\text { Finasterida } \\
\text { Study } \\
\text { Group }\end{array}$ & $\begin{array}{l}\text { Doble ciego } \\
\text { controlado } \\
\text { Multicéntrico } \\
\text { aleatorizado }\end{array}$ & $\begin{array}{l}1100 \\
\text { pacientes }\end{array}$ & $\begin{array}{l}1 \text { y } 5 \mathrm{mg} \\
\text { finasterida/placebo } \\
12 \text { meses + extensión } \\
\text { de } 4 \text { años todos en } \\
\text { tratamiento }\end{array}$ & $\begin{array}{l}\text { Health related quality } \\
\text { of life } \\
\text { (cuestionario no } \\
\text { especifico) }\end{array}$ & $\begin{array}{l}\text { Mejoría significativa } \\
\text { de los sintomas } \\
\text { clinicos. } \\
\text { El tratamiento } \\
\text { incrementa la } \\
\text { puntuación media de } \\
\text { la esfera sexual } \\
\text { Escasos cambios en } \\
\text { otros aspectos de } \\
\text { calidad de vida } \\
\text { En un pequeño } \\
\text { porcentaje empeora la } \\
\text { función sexual } \\
\text { Los efectos se } \\
\text { mantienen en el } \\
\text { periodo de extensión }\end{array}$ & II & 3 \\
\hline $\begin{array}{l}\text { Lowe }^{34} \\
1995\end{array}$ & $\begin{array}{l}\text { Evaluación } \\
\text { económica }\end{array}$ & $\begin{array}{l}\text { Árbol de } \\
\text { decisión }\end{array}$ & $\begin{array}{l}\text { Terazosin vs. Cirugia } \\
\text { vs. Finasterida } \\
\text { durante los dos } \\
\text { primeros años }\end{array}$ & $\begin{array}{l}\text { Análisis de costes, } \\
\text { duración de la } \\
\text { mejoria, pérdida de } \\
\text { días productivos }\end{array}$ & $\begin{array}{l}\text { La intervención más } \\
\text { costosa es la cirugía y } \\
\text { la más económica la } \\
\text { terazosina estimado } \\
\text { para dos años. La } \\
\text { duración de la } \\
\text { mejoría es } \\
\text { comparable en los } \\
\text { tres }\end{array}$ & & \\
\hline $\begin{array}{l}\text { Balad }{ }^{35} \\
1996\end{array}$ & $\begin{array}{l}\text { Evaluación } \\
\text { económica }\end{array}$ & $\begin{array}{l}\text { Coste } \\
\text { efectividad/coste } \\
\text { utilidad }\end{array}$ & $\begin{array}{l}\text { RTU vs. observación } \\
\text { vs. finasterida }\end{array}$ & $\begin{array}{l}\text { Probabilidad de } \\
\text { reducción de la } \\
\text { severidad de los } \\
\text { sintomas } \\
\text { Coste por QALY } \\
\text { 3000-97000\$Can }\end{array}$ & $\begin{array}{l}\text { Finasterida es la } \\
\text { alternativa dominante } \\
\text { en pacientes con } \\
\text { sintomas moderaods } \\
\text { y tratamiento <3 } \\
\text { años, pero no cuando } \\
\text { los sintomas son } \\
\text { severos }\end{array}$ & & \\
\hline $\begin{array}{l}\text { Albertsen }{ }^{36} \\
1999\end{array}$ & $\begin{array}{l}\text { Evaluación } \\
\text { económica }\end{array}$ & $\begin{array}{l}\text { Minimización } \\
\text { de costes }\end{array}$ & $\begin{array}{l}\text { Terazosin vs. } \\
\text { observación vs. } \\
\text { finasterida }\end{array}$ & $\begin{array}{l}\text { Análisis de costes } \\
\text { según valores PSA } \\
\text { en evitar eventos } \\
\text { obstructivos y cirugia } \\
\text { obstructivos y cirugia } \\
\text { prostática }\end{array}$ & $\begin{array}{l}\text { Finasterida es más } \\
\text { económico que otros } \\
\text { tratamientos en } \\
\text { pacientes con grandes } \\
\text { próstatas }\end{array}$ & & \\
\hline
\end{tabular}


TABLA IV (Continuación)

CARACTERIZACIÓN DE LOS ESTUDIOS REVISADOS

\begin{tabular}{|c|c|c|c|c|c|c|c|}
\hline Autor & Diseño & Población & Intervención & Resultados & Conclusiones & $\begin{array}{l}\text { Nivel de } \\
\text { evidencia }\end{array}$ & $\begin{array}{l}\text { Calidad } \\
\text { ECC }\end{array}$ \\
\hline $\begin{array}{l}\text { Kaplan }^{37} \\
1996\end{array}$ & $\begin{array}{l}\text { Satisfacción } \\
\text { del paciente }\end{array}$ & 211 pacientes & $\begin{array}{l}\text { Finasterida durante 9- } \\
12 \text { meses } \\
\text { Cuestionario } \\
\text { telefónico }\end{array}$ & $\begin{array}{l}\text { Severidad de los } \\
\text { sintomas } \\
\text { Ocurrencia de los } \\
\text { sintomas } \\
\text { Respuesta global al } \\
\text { tto } \\
\text { Grado de mejoría }\end{array}$ & $\begin{array}{l}78 \% \text { los sintomas } \\
\text { urinarios no } \\
\text { interferian sus } \\
\text { actividades } \\
54 \% \text { subjetivamente } \\
\text { su salud era excelente } \\
\text { o muy buena } \\
87 \% \text { encontraron } \\
\text { mejoría sintomática }\end{array}$ & & \\
\hline $\begin{array}{l}\text { Bruskewitz }^{38} \\
1999 \\
\text { PLESS }\end{array}$ & $\begin{array}{l}\text { Doble ciego } \\
\text { controlado } \\
\text { Multicéntrico } \\
\text { Aleatorizado }\end{array}$ & $\begin{array}{l}3040 \\
\text { pacientes }\end{array}$ & $\begin{array}{l}5 \mathrm{mg} \text { Finasterida vs } \\
\text { placebo }\end{array}$ & $\begin{array}{l}\text { BII } \\
\text { AUASI }\end{array}$ & $\begin{array}{l}\text { Finasterida mejora la } \\
\text { sintomatologia } \\
\text { urinaria más evidente } \\
\text { en pacientes cuyo } \\
\text { PSA >1,4 ng/ml } \\
\text { La satisfacción y } \\
\text { conducta sexual } \\
\text { empeora ligeramente } \\
\text { en pacientes con PSA } \\
<1,4 \text { ng/ml pero } \\
\text { escasa diferencia en > } \\
\text { CVRS mejora } \\
\text { sobre todo en } \\
\text { pacientes con PSA } \\
>1,4 \mathrm{ng} / \mathrm{ml}\end{array}$ & II & 3 \\
\hline
\end{tabular}

seleccionar los pacientes, donde Finasterida va a lograr mayor respuesta. Sólo en aquellos con próstata mayores de $40 \mathrm{ml}$ la respuesta fue significativa con una reducción media del IPSS en 2,8 y un aumento del FUM de $1,84 \mathrm{ml} / \mathrm{seg}$.

Andersen et al. ${ }^{21}$ en su meta-análisis que incluye 4.222 pacientes con un seguimiento mínimo de 2 años, demuestra que Finasterida reduce en un $57 \%$ el riesgo de retención aguda de orina y un $34 \%$ el de cirugía, comparado con placebo.

Roehrborn $^{24}$ en 1998, confirma de nuevo el valor del volumen prostático para predecir la respuesta al tratamiento con Finasterida. Lo mismo sucede con el PSA, ya que los varones con valores de inicio mayor de 1,4 ngr/ml respondieron mejor al fármaco.

La relación entre volumen prostático y PSA, es clara a través de la mayor distribución de tejido epitelial en las próstatas de mayor tamaño, lo que propicia una mayor secreción de PSA. Por tanto, estos dos parámetros, volumen y PSA, pero especialmente el primero, han de ser considerados a la hora de elegir el fármaco para de esta forma incrementar las posibilidades de respuesta, ya que los pacientes con próstatas pequeñas $(<40 \mathrm{ml})$ o bajos valores de PSA $(<1,4 \mathrm{ngr} / \mathrm{ml})$, tienen escasas posibilidades de beneficiarse con el uso de Finasteride.

En los estudios doble ciego, controlados y multicéntricos aleatorizados (nivel II), se observan los siguientes hallazgos: Finasterida reduce el volumen prostático, una media del $20 \%$. La reducción máxima se obtiene a partir del $6^{\circ}$ mes de tratamiento y se mantiene a lo largo del seguimiento. Ello significa que se modifica la evolución natural de la hiperplasia, que tiende a crecer de forma continuada, en mayor o menor medida. Los síntomas obstructivos mejoran, especialmente el FUM y el residuo post-miccional. Los pacientes que de inicio tienen sintomatología débil y moderada, consiguen el mayor beneficio sintomatológico. Sin embargo, en el estudio de Byrnes et al. ${ }^{11}$ también mejoran aquellos con sintomatología severa. Se confirma la reducción del riesgo de retención aguda de orina y de cirugía. En cualquier caso, la mejoría sintomática es de inicio tardío (más de tres meses) y progresiva, manteniéndose a largo plazo. Hay estudios con seguimiento superior a siete años ${ }^{19}$. 


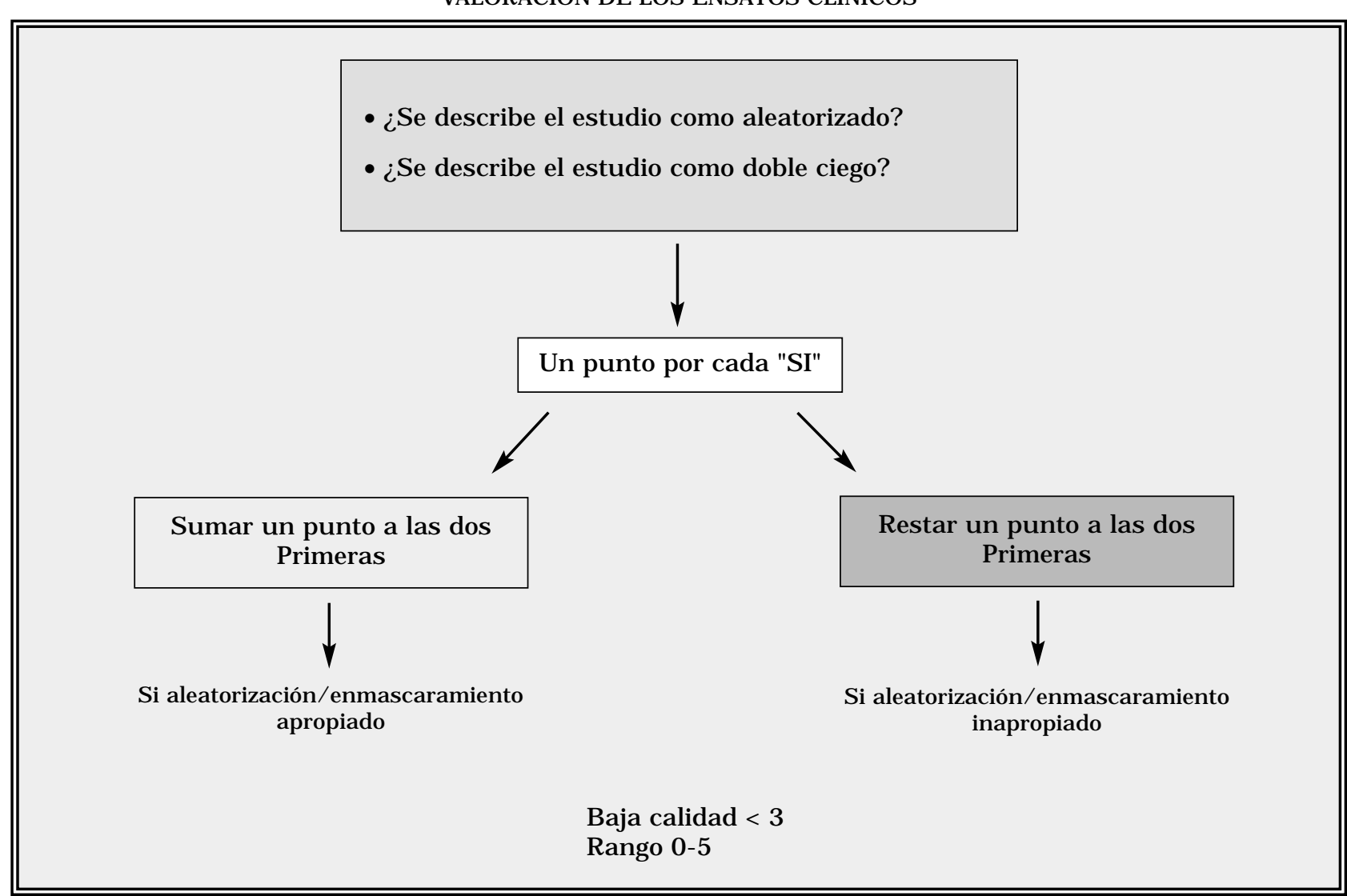

(Jaddad AR et al., 1996)

Los mayores beneficios de Finasterida se alcanzan en las próstatas con un volumen mayor de $40 \mathrm{ml}$.

El uso de Finasterida comporta un bajo número de efectos adversos, que se centran mayoritariamente en la esfera sexual, sobre todo durante el primer año del tratamiento. Sin embargo el porcentaje de abandonos, por esta causa, es bajo, alrededor del $2 \%$.

Tres estudios comparan Finasterida con otros fármacos. Lepor et al. ${ }^{18}$ en un estudio multicéntrico aleatorizado simple ciego, incluye 1.229 pacientes distribuidos en 4 brazos. Finasterida (F), Terazosina $(T), T+F$, placebo, seguidos 52 semanas.

El estudio demuestra mayor eficacia de T, siendo el efecto de Finasterida similar a placebo. El tratamiento combinado de $\mathrm{T}+\mathrm{F}$, no mejora los resultados obtenidos con $\mathrm{T}$ sola. La crítica a este estudio reside en el gran número de pacientes con próstatas pequeñas $(<40 \mathrm{ml})$ que participan en el estudio y que podría justificar los pobres resultados obtenidos con $\mathrm{F}$.

Sokeland et al. ${ }^{31}$ comparan Finasterida con Seronoa repens y Urtica (extractos de plantas). Con un seguimiento de 48 semanas, no encontraron diferencias en la eficacia entre ambos fármacos, mientras que la tolerancia fue mejor con los extractos de planta. La respuesta fue independiente del volumen prostático.

Finalmente Debruyne et al. ${ }^{26}$ realizan un estudio doble ciego multicéntrico, aleatorizado entre Alfuzosina y Finasterida y la combinación de ambos. La respuesta a Alfuzosina fue mayor que a Finasterida, sin que la combinación mejorara los resultados obtenidos con Alfuzosina, sola.

Dos estudios valoran el efecto de Finasterida sobre la obstrucción. Mediante estudio de presión/flujo, Abrams et al. ${ }^{27}$ observaron que Finasterida provocaba una disminución significa- 
tiva de la presión del detrusor a máximo flujo. En los pacientes con próstatas mayores de $40 \mathrm{ml}$ la reducción fue mayor, que en aquellos con próstatas más pequeñas, aunque las diferencias no fueron significativas. De nuevo, esta vez con un estudio objetivo, se demuestra una mayor respuesta a Finasterida, en las próstatas más grandes, donde la reducción volumétrica se acompaña de una disminución de los parámetros obstructivos, demostrándose así un efecto fisiopatológico claro.

Estos datos fueron confirmados en un seguimiento a dos años, donde persistía la mejoría presión/flujo ${ }^{29}$.

En los estudios económicos se constata que para un período de dos años de seguimiento, la RTU de próstata es la opción más cara, seguido a gran distancia de Finasterida y Terazosina (641), 2860, 2422 dólares, respectivamente), todo ello con una mejoría sintomática de 88,67 y $74 \%$ para cada uno de aquellos tratamientos ${ }^{34}$. Por tanto sería necesario un período de tratamiento continuado de al menos 5 años, para que se igualen los costes de la terapia farmacológica y quirúrgica. Todo ello sin tener en cuenta las repercusiones en la calidad de vida, de las opciones.

De otra parte, el fallo del tratamiento farmacológico con cirugía secundaria, representaría el peor escenario posible, desde el punto de vista económico.

Albertesen et al. ${ }^{36}$ utilizan un modelo analítico de decisión y analizan el coste de esperar (placebo), tratamiento con Finasterida, Terazosina o RTU, basándose en la incidencia de retención aguda de orina o necesidad de cirugía, teniendo en cuenta lo esperado en la evolución natural o los datos ofrecidos por el estudio PLESS (tratamiento con Finasterida, durante cuatro años) y el estudio HYCAT (tratamiento con Terazosina, durante un año).

Para dos años de seguimiento, el máximo coste fue para la RTU (8966 dólares) el menor para esperar y ver (1802 dólares). Finasterida fue 240 dólares más barato que Terazosina. Las diferencias fueron mayores en los pacientes con PSA inicial, $>1,4 \mathrm{ng} / \mathrm{ml}$, que tienen próstatas mayores.

En un estudio realizado en Canadá ${ }^{35}$ con tres años de seguimiento, Finasterida fue más costeefectivo que esperar y ver, por la reducción en retenciones y cirugía y que RTU, en pacientes moderadamente sintomáticos.
Utilizando cuestionarios para evaluar la calidad de vida relacionada con la salud y la enfermedad específica (HRQOL). Bruskewitz et al. ${ }^{38}$ analizan los 3.040 pacientes incluidos en el estudio PLESS y que recibieron Finasterida o placebo, durante cuatro años. A partir de los cuatro meses de inicio del estudio, aquellos tratados con Finasterida mejoran sus síntomas lo que implica una reducción significativa de la interferencia de los mismos con la actividad diaria y de la preocupación por dicha sintomatología. Las diferencias en calidad de vida, fueron mayores en aquellos que tenían PSA $>1,4$ $\mathrm{ng} / \mathrm{ml}$. La satisfacción y el deseo sexual fueron ligeramente peores en el grupo de Finasterida que en el de placebo, aunque los pacientes con PSA >1,4 $\mathrm{ng} / \mathrm{ml}$, no refieren diferencias.

Otro estudio ${ }^{37}$ también sugiere que Finasterida mejora la calidad de vida relacionada con la enfermedad. Teniendo en cuenta la alta incidencia de síntomas de origen prostático en la población mayor de 60 años, que además por el envejecimiento de la misma, es cada vez mayor, esta mejoría en la calidad de vida tiene una gran repercusión individual y social.

\section{CONCLUSIONES}

$\mathrm{El}$ análisis de los estudios realizados demuestra, con alto nivel de evidencia (I-II-III), que Finasterida modifica la evolución natural de la hiperplasia prostática benigna al reducir su volumen, lo que lleva implícito una significativa mejoría sintomática, que se mantiene en el tiempo y que es especialmente llamativo en la reducción del riesgo de retención aguda de orina y cirugía.

Estos beneficios son más frecuentes en las próstatas de mayor volumen ( $>40 \mathrm{ml}$ ).

Con estudios presión/flujo se evidencia que la reducción de tamaño comporta mejoría en el patrón obstructivo miccional.

Únicamente tres estudios comparan Finasterida con otros fármacos (Terazosina, Alfuzosina, Extractos de plantas). Los alfabloqueantes consiguen mejor respuesta clínica que Finasterida, con un seguimiento máximo de un año.

Desde el punto de vista económico, el uso de Finasterida y Terazosina es más coste-efectivo que la RTU de próstata, con un seguimiento de dos años. 
Finasterida mejora la calidad de vida de los pacientes con sintomatología dependiente de la hiperplasia prostática benigna.

En consecuencia Finasterida después de más de 10 años de empleo en la clínica diaria ha superado la prueba del tiempo mostrándose como un fármaco de eficacia duradera y con escasos efectos adversos.

El nivel de calidad de las evidencias científicas encontradas, permite un grado de recomendación bueno (categoría A), para pacientes con próstatas mayores de $40 \mathrm{ml}$, donde la mejoría sintomática y los escasos efectos adversos, repercute en la calidad de vida (grado de recomendación A).

\section{REFERENCIAS}

1. JOVELL JA, NAVARRO RUBIO MD.: Evaluación de la evidencia científica. Med Clin 1995; 105: 26-29.

2. JADDAD AR, MOORE RA, CARROLL D, JENKINSON C, RENOLDS JM, GAVAGHAN DJ et al.: Assesing the quality of reports on randomized clinicals trials: is blinding necessary? Controlled Clin Trials 1996; 17: $1-12$.

3. BEISLAND HO, BINKOWITZ B, BREKKAN E, EKMAN P, KONTTURI M, LEHTONEN T et al.: Scandinavian clinical study of finasteride in the treatment of benign prostatic hyperplasiaa. European Urology 1992; 22 (4): 271-277.

4. KIRBY RS BJ.: Finasteride in the treatment of benign prostatic hyperplasia. A urodynamic evaluation. British Journal of Urology 1992; 70 (1): 65-72.

5. GORMLEY GJ, STONER E, BRUSKEWITZ RC, IMPERATO-McGINLEY J, WALSH PC, MCCONNELL JD et al.: The effect of finasteride in men with benign prostatic hyperplasia. The Finasteride Study Group. [see comments]. New England Journal of Medicine 1992; 327 (17): 1185-1191.

6. STONER E.: The clinical effects of a 5 alpha-reductasa inhibitor, finasteride, on benign prostatic hyperplasia. The Finasteride Study Group. Journal of Urology 1992; 147 (5): 1298-1302.

7. KIRBY RS VJ.: Long-term urodynamic effects of finasteride in benign prostatic hyperplasia: a pilot Study. European Urology 1993; 24 (1): 20-26.

8. EKMAN P.: Finasteride in the treatment of benign prostatic hypertrophy. Journal D'Urologie 1993; 99 (6): 299-302.

9. STONER E.: Three-year safety and efficacy data on the use of finasteride in the treatment of benign prostatic hyperplasia. Urology 1994; 43 (3): 284292.

10. ANDERSEN JT, EKMAN P, WOLF H, BEISLAND HO, JOHANSSON JE, KONTTURI $M$ et al.: Can finasteride reverse the progress of benign prostatic hyperplasia? A two-year placebo-controlled study. The Scandinavian BPH Study Group. Urology 1995; 46 (5): 631-637.
11. BYRNESS CA, MORTON AS, LISS CL, LIPPERT MC, GILLENWATER JY.: Efficacy, tolerability, and effect on health-related quality of life of finasteride versus placebo in men with symptomatic benign prostatic hyperplasia: a community based study. CUSP Investigators. Community based study of Proscar. Clinical Therapeutics 1995; 17 (5): 956969.

12. GELLER J.: Five-year follow-up of patients with benign prostatic hyperplasia treated with finasteride. European Urology 1995; 27 (4): 267-273.

13. TAMMELA TL KM.: Long-term effects of finasteride on invasive urodynamics and symptoms in the treatment of patients with bladder outflow obstruction due to benign prostatic hyperplasia. Journal of Urology 1995; 154 (4): 1466-1469.

14. MOORE E.: Proscar: five-year experience [published erratum appears in Eur Urol 1996; 29 (2): 234]. European Urology 1995; 28 (4): 304-309.

15. NICKEL JC, FRADET Y, BOAKE RC, POMMERVILLE PJ, PERREAULT JP, AFRIDI SK et al.: Efficacy and safety of finasteride therapy for benign prostatic hyperplasia: results of a 2-year randomized controlled trial (the PROSPECT Study). CMAJ: Canadian Medical Association Journal 1996; 155 (9): 12511259.

16. BOYLE P, GOULD AL, ROEHRBORN CG.: Prostate volume predicts outcome of treatment of benign prostatic hyperplasia with finasteride: meta-analysis of randomized clinical trials. Urology 1996; 48 (3): 398-405.

17. CARRARO JC, RAYNAUD JP, KOCH G, CHISHOLM GD, DI SILVERIO F, TEILLAC P et al.: Comparison of phytotherapy (Permixon(TM) with finasteride in the treatment of benign prostate hyperplasia: a randomized international study of 1,098 patients. Prostate 1996; 29 (4): 231-240.

18. LEPOR H.: The efficacy of terazosin, finasteride, or both in benign prostatic hyperplasia. New England Journal of Medicine 1996; 335 (8): 533-539.

19. MARTÍNEZ SARMIENTO M, CUÑAT ALBERT E, LÓPEZ ALCINA E, PONTONES MORENO JL, SANZ CHINESTA S, JIMÉNEZ CRUZ JF.: Tratamiento de la hiperplasia benigna de próstata con finasteride. Resultados a 7 años. Actas Urológicas Españolas 1997; 2: 105-110.

20. TENOVER JL, PAGANO GA, MORTON AS, LISS CL, BYRNES CA.: Efficacy and tolerability of finasteride in symptomatic benign prostatic hyperplasia: a primary care study. Clinical Therapeutics 1997; 19 (2): 243-258.

21. ANDERSEN JT, NICKEL JC, MARSHALL VR, SCHULMAN CC, BOYLE P.: Finasteride significantly reduces acute urinary retention and need for surgery in patients with symptomatic benign prostatic hyperplasia. Urology 1997; 49 (6): 839-845.

22. KUO HC.: Comparative study of therapeutic effect of dibenyline, finasteride, and combination drugs for symptomatic benign prostatic hyperplasia. Urologia Internationalis 1998; 60 (2): 85-91.

23. EKMAN P.: Maximum efficacy of finasteride is obtained within 6 months and maintained over 6 years. Follow-up of the Scandinavian Open-Extension Study. European Urology 1998; 33 (3): 312-317. 
24. ROEHRBORN CG.: Meta-analysis of randomized clinical trials of finasteride. Urology 1998; 51 supl: 469.

25. MARBERGER MJ.: Long-term effects of finasteride in patients with benign prostatic hyperplasia: a double-blind, placebo-controlled, multicenter study. PROWESS Study Group. Urology 1998; 51 (5): 677 686.

26. DEBRUYNE FMJ, JARDIN A, COLLOI D, RESEL L, WITJES WPJ, DELAUCHE-CAVALLIER MC et al.: Sustained-release alfuzosin, finasteride and the combination of both in the treatment of benign prostatic hyperplasia. European Urology 1998; 34 (3): 169-175.

27. ABRAMS P.: Improvement of pressure flow parameters with finasteride is greater in men with large prostates. Finasteride urodynamics study group. Journal of Urology 1999; 161 (5): 1513-1517.

28. HUDSON PB BR.: Efficacy of finasteride is maintained in patients with benign prostatic hyperplasia treated for 5 years. The North American Finasteride Study Group. Urology 1999; 53 (4): 690-695.

29. SCHAFER W, TAMMELA TJL, BARRETT DM, ABRAMS $P$ et al.: Continued improvement in preassure-flow parameters in men receiving finasteride for two years. Urology 1999; 54: 278-282.

30. MARKS LS, PARTIN AW, DOREY FJ et al.: Long term effects of finasteride on prostate tissue composition. Urology 1999; 53 (3): 574-580.

31. SOKELAND K.: Combined sabal and urtica extract compared with finasteride in men with benign prostatic hyperplasia: analysis of prostate volume and therapeutic outcome. BJU International 2000; 86: 439-442.

32. KAPLAN SA, HOLTGREWE HL, BRUSKEWITZ R, SALTZMAN B, MOBLEY D, NARAYAN P et al.: Comparison of the efficacy and safety of finasteride in older versus younger men with benign prostatic hyperplasia. Urology 2001; 57 (6): 1073-1077.
33. GIRMAN CJ, KOLMAN C, LISS CL, BOLOGNESE JA, BINKOWITZ BS, STONER E.: Effects of finasteride on health-related quality of life in men with symptomatic benign prostatic hyperplasia. Finasteride Study Group. Prostate 1996; 29 (2): 83-90.

34. LOWE FC, McDANIEL RL, CHMIEL JJ, HILLMAN AL.: Economic modeling to assess the costs of treatment with finasteride, terazosin, and transurethral resection of the prostate for men with moderate to severe symptoms of benign prostatic hyperplasia. [see comments]. Urology 1995; 46 (4): 477-483.

35. BALADI JF, MENON D, OTTEN N.: An economic evaluation of finasteride for treatment of bening prostatic hyperplasia. Pharmacoeconomics 1996; 9: 443454.

36. ALBERTESEN PA, PELLISSIER JM, LOWE F, GIRMAN CJ, ROEHRBORN CG.: Economic analysis of finasteride: a model-based approach using data from the proscar long term efficacy and safety studt. Clinical Therapeutics 1999; 21: 1006-1024.

37. KAPLAN SA, OLSSON CA.: Patient satisfaction with finasteride in the treatment of symptomatic benign prostatic hyperplasia. Clinical Therapeutics 1996; 18 (1): 73-83.

38. BRUSKEWITZ R, GIRMAN CJ, FOWLER J, RIGBY OF, SULLIVAN M, BRACKEN RB et al.: Effect of finasteride on bother and other health-related quality of life aspects associated with benign prostatic hyperplasia. Urology 1999; 54 (4): 670-678.

Dr. J.F. Jiménez Cruz

Servicio de Urología

Hospital Universitario La Fe

Avda. Campanar, 21

46009 Valencia

(Trabajo recibido el 20 septiembre de 2002) 